\title{
A Novel Super-resolution Approach Based on Supervised Canonical Correlation Analysis
}

\author{
Suna Xia, Gangmin Zheng, Yuanyuan Ma and Xiaohu Ma \\ Department of Computer Science and Technology, Soochow University, 215006, Suzhou, Jiangsu Province, China \\ 20104227025@suda.edu.cn,20104227036@suda.edu.cn,20104227039@suda.edu.cn,xhma@suda.edu.cn
}

\begin{abstract}
In this paper, we use supervised canonical correlation analysis (SCCA) method to extract features which maximize the correlation between HR and LR face images. Then Relationship Learning (RL) is used to construct the mapping relationship between the face coherent features. SCCA method comprehensively considers the within-class information and the similarity of HR and LR images, to make the SR image closer to original HR image. Experiments on Yale and ORL face databases show that our method has higher recognition rate.

Index Terms - Super-resolution, High Resolution, Low Resolution, Supervised Canonical Correlation Analysis, Relationship Learning
\end{abstract}

\section{Introduction}

The purpose of face recognition is to recognize the identity of the person through extracting the discrimination information from the face images. Super-resolution[1] refers to getting a clear high resolution(HR) image from one or multiple low resolution(LR) images through feature extraction and reconstruction. Now there are three major super resolution methods. Among them, the learning method is the hot spot of the current super resolution technology research.

In recent years, CCA has been widely used for data analysis. At the same time, more and more improved CCA methods have appeared. Sub-pattern CCA(SpCCA)[2] uses the correlation properties between global and local characteristic vectors as effective discrimination information. Because CCA and least square has a certain similarity in multi-label classifications, Sun[3] proposes the least-square CCA(LSCCA) method. Hou Shu-dong[4] proposes a supervised locality preserving CCA(SLPCCA) algorithm, which effectively inherits the advantages of CCA and LPP.

In this paper, we will use the supervised canonical correlation analysis(SCCA) method, which brings classification information into the traditional CCA to preserve local information. Comparing with SLPCCA, SCCA pays more attention to maximize the correlation between HR and LR images, but SLPCCA prefers to preserve the local manifold structure of the data. In our algorithm, relationship learning(RL)[5] will be used to estimate mapping matrix between HR images and LR images.

\section{Background and Related Works}

In this section, we will introduce CCA, SCCA method, and analysis them.

Related works. CCA method projects variables into the coherent subspaces in which the correlation is maximize.
However, there are several limitations with CCA. The first is small sample size with high dimension problem. In this paper, we will use PCA to avoid this problem. The second is the lack of application of the local information, which will affects the recognition rate especially for local variants. To overcome this problem, SCCA uses similarity matrix including the class label information. The SCCA method protects intrinsic structures of HR and LR face images.

SCCA effectively inherits the advantage of CCA and adopts weight relation which is based on the class label information. Relationship learning is used to build a transformation between HR and LR face features, and the relationship matrix will be used to construct the SR images. So the SCCA\&RL method will be more useful to SR reconstruction in face recognition.

Supervised Canonical Correlation Analysis. HR face image and its corresponding LR face image have the similarity intrinsic geometries, and in coherent subspaces, they have the statistical correlation. Huang Hua[6,7] proposes superresolution methods of face image using $\mathrm{CCA}$ and RBF. Yueting Zhuang[8] proposes LPH super-resolution and neighbor reconstruction for residue compensation. In this paper, our method not only effectively extracts related features, but also to protect the local manifold, so in the field of SR research, the robustness of SCCA will be better.

Unlike the traditional CCA method, SCCA constructs the similarity matrix $\mathrm{W}^{\mathrm{H}}$ and $\mathrm{W}^{\mathrm{L}}$ first. Given two zero-mean vector sets $X^{H}=\left\{x_{1}^{h}, x_{2}^{h}, \ldots, x_{m}^{h}\right\}$ and $X^{L}=\left\{x_{1}^{l}, x_{2}^{l}, \ldots, x_{m}^{l}\right\}$,

$W^{H}(i, j)=\left\{\begin{array}{c}\exp \left(-\frac{\left\|x_{i}^{h}-x_{j}^{h}\right\|^{2}}{t^{h}}\right), \text { if } \mathrm{l}\left(x_{i}^{h}\right)=\mathrm{l}\left(x_{j}^{h}\right), \\ 0, \text { othersize }\end{array}\right.$

$W^{L}(i, j)=\left\{\begin{array}{c}\exp \left(-\frac{\left\|x_{i}^{l}-x_{j}^{l}\right\|^{2}}{t^{l}}\right), \text { if } 1\left(x_{i}^{l}\right)=\mathrm{l}\left(x_{j}^{l}\right)(1) \\ 0, \text { othersize }\end{array}\right.$

$1(\bullet)$ means the class. Then we will obtain new feature samples $\widehat{X}^{H}=X^{H} W^{H}$ and $\widehat{X}^{L}=X^{L} W^{L}$.

Using the new sets of features and traditional CCA algorithm, we will find a pair of projection directions $\mathrm{U}$ and $\mathrm{V}$, 
which maximize the correlation of $X=U^{T} \widehat{X}^{H}$ and $Y=V^{T} \hat{X}^{L}$. The correlation coefficient will be represented by $\rho$, then our objective function is

$\max \rho=\frac{E\left[X Y^{T}\right]}{\sqrt{E\left[X X^{T}\right] \cdot E\left[Y Y^{T}\right]}}=\frac{U^{T} E\left[\widehat{X}^{H}\left(\hat{X}^{L}\right)^{T}\right] V}{\sqrt{U^{T} E\left[\widehat{X}^{H}\left(\hat{X}^{H}\right)^{T}\right] U \cdot V^{T} E\left[\hat{X}^{L}\left(\hat{X}^{L}\right)^{T}\right] V}}$

\section{Super-resolution Algorithm based on SCCA and Relationship Learning}

In this section, we will introduce the details of our SCCA\&RL algorithm. There are four steps to train face images. First of all, we choose PCA to extract training features. Secondly, SCCA will be used to extract the coherent subspaces. This step consists of three processes, construction of similarity matrix within-class, obtaining base vectors which maximize the correlation coefficients between HR and LR face images, and projecting the coefficients of PCA models into the coherent subspaces which SCCA achieves. Thirdly, we use relationship learning to build a transformation between HR and LR face images. At last, a NN classifier is used for recognition result. LR testing face images contain three steps, PCA feature extraction, the projection with the base vectors of LR training images, and comparing Euclidean distance with LR training data in SCCA subspace.

Given a set of HR training face images, $\mathrm{I}^{\mathrm{H}}$, then we obtain the LR training face images $I^{\mathrm{L}}$ through down-sampling HR training images. After PCA feature extraction, we get new zero-mean datasets $X^{H}=\left\{x_{1}^{h}, x_{2}^{h}, \ldots, x_{m}^{h}\right\} \in \mathfrak{R}^{p * m} \quad$ and $X^{L}=\left\{x_{1}^{l}, x_{2}^{l}, \ldots, x_{m}^{l}\right\} \in \mathfrak{R}^{q^{* m}}, \mathrm{~m}$ denotes the size of HR training images. SCCA is used to obtain the coherent subspaces to make the new datasets related.

SCCA Projection. As mentioned above, we construct the similarity matrix $\mathrm{W}^{\mathrm{H}}$ and $\mathrm{W}^{\mathrm{L}}$ first. Then $\widehat{X}^{H}=X^{H} W^{H}$ and $\widehat{X}^{L}=X^{L} W^{L}$ are used to capture the coherent subspaces as the CCA method. CCA determines the subspaces with two base vectors $\mathrm{U}$ and $\mathrm{V}$, to maximize the correlation between $C^{H}=U^{T} \widehat{X}^{H}$ and $C^{L}=V^{T} \widehat{X}^{L}$.

To solve Eq. 2, we define $R_{H H}=E\left[\widehat{X}^{H}\left(\widehat{X}^{H}\right)^{T}\right]$ and $R_{L L}=E\left[\widehat{X}^{L}\left(\widehat{X}^{L}\right)^{T}\right]$ as the within-class covariance matrices of $\hat{X}^{H}$ and $\hat{X}^{L}, R_{H L}=E\left[\hat{X}^{H}\left(\hat{X}^{L}\right)^{T}\right]$ and $R_{L H}=E\left[\hat{X}^{L}\left(\hat{X}^{H}\right)^{T}\right]$ as between-class covariance matrices. Then we get two matrixes

$$
R_{1}=R_{H H}^{-1} R_{H L} R_{L L}^{-1} R_{L H}, R_{2}=R_{L L}^{-1} R_{L H} R_{H H}^{-1} R_{H L}
$$

When the eigenvalues of $R_{1}$ and $R_{2}$ are in the descending order, $\mathrm{U}$ and $\mathrm{V}$ are the corresponding eigenvectors. Here, we obtain the SCCA coherent subspaces for HR and LR face images.

$$
\begin{aligned}
& C^{H}=U^{T} \widehat{X}^{H}, C^{H}=\left\{c_{1}^{h}, c_{2}^{h}, \ldots, c_{m}^{h}\right\} \in \mathfrak{R}^{y^{*} m}, \\
& C^{L}=V^{T} \widehat{X}^{L}, C^{L}=\left\{c_{1}^{l}, c_{2}^{l}, \ldots, c_{m}^{l}\right\} \in \mathfrak{R}^{z^{*} m}
\end{aligned}
$$

Among these, $y$ and $z$ denotes the dimensions in SCCA subspace of HR and LR face images.

Relationship Learning. Relationship learning is to build a transformation between HR and LR face features in their coherent subspaces. In some papers, they use RBF to construct the mapping relationship. Comparing with our method, it is useful to construct SR images. But when we calculate the recognition rate, our method will perform better. Here is the objective function

$$
\min _{R} \varepsilon(R)=\frac{1}{m} \sum_{i=1}^{m}\left(\left\|c_{i}^{h}-R c_{i}^{l}\right\|^{2}\right)+\beta\|D R-I\|^{2}
$$

Where $\mathrm{R}$ is the relationship matrix, $\Sigma(\bullet)$ is the construction error obtained by Euclidean distance, $\|\bullet\|$ is Frobenius norm, $\beta$ is the normalization, D is downsampling matrix and $I$ is the identity matrix.

Gradient descent is used to minimize Eq. 5, and matrix $\mathrm{R}$ is achieved with n-th iteration, $R^{(k)}=R^{(k-1)}-\alpha \frac{\partial \varepsilon(R)^{T}}{\partial R}$, where $\alpha$ is the length of descent. To save the computing time, we set $\alpha=1 /\left(R^{(1)}-R^{(0)}\right)$ in our experiment. When the difference between $R^{(n)}$ and $R^{(n-1)}$ is very small, $R^{(n)}$ will be the relationship matrix $\mathrm{R}$ we want.

Supposing that there is a LR test face image, and $t$ is the feature extracted by PCA. Then we project it into the coherent subspace,

$$
c_{1}=V^{T}\left(t-\mu^{l}\right), \quad \tilde{c}_{h}=R c_{1}
$$

$\mu^{l}$ is the mean feature of LR training images after PCA extraction, $c_{1}$ is the feature extracted by SCCA. Using the relationship matrix $\mathrm{R}, \tilde{c}_{h}$ is the SR feature we calculate.

At last, we compute the Euclidean distance between $\tilde{c}_{h}$ and every vector in matrix $C^{L}$. Choosing the vector which has the minimum distance with $\tilde{c}_{h}$, we think it and the testing vector belong to the same person.

\section{Experiments and Discussion}

In these experiments, we use Yale and ORL face databases. Yale face database obtains 15 persons. Each one has $11 \mathrm{HR}$ face images with size $32 * 32$. These images are down-sampled to LR face images of size $8 * 8$ as shown in Fig.1. Images in ORL and Yale face databases have the same size, so does the LR images. The language experiments used is MATLAB. 
In our experiments, we mainly discuss two questions. The first is the influence on recognition rate with different dimensions extracted. The other question is the influence on recognition rate with different number of training images. To show the validity of our algorithm, we compare our method with three different methods, CCA\&RL, LPH\&RBF[8], and CCA\&LPH is a two-step SR method.

In Fig.1, we compare our method with other methods. These face images show that CCA is good at capturing the global information and LPP performances well at the local position. As a whole, our method has the best performance among these methods.

(a)
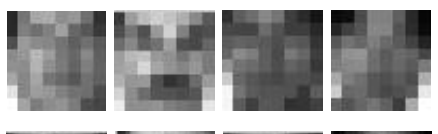

(b)
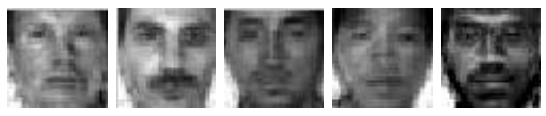

(c)
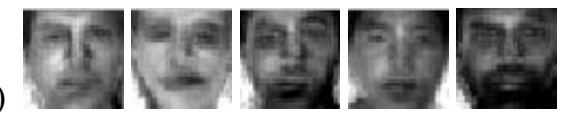

(d)
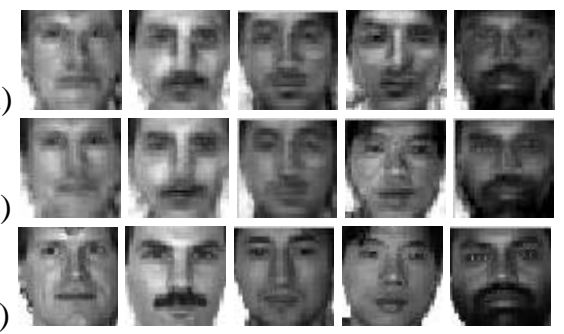

Fig.1. Comparing our method with other three methods. (a) LR face images; (b) CCA\&RL; (c) LPH\&RBF; (d) CCA\&LPH; (e) SR face images based on our method; (f) Original HR face images.

Experiments of Yale face database. In this face database, the first six face images are training samples, and the other five face images are testing images.

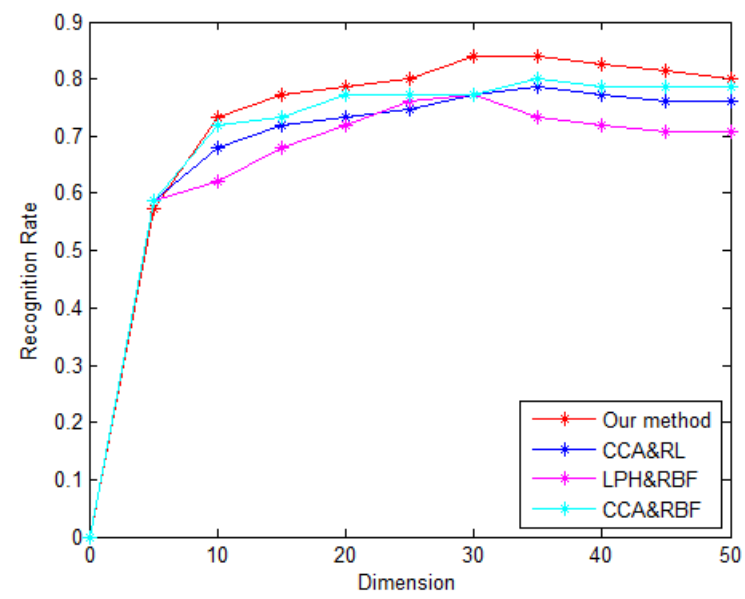

(a)

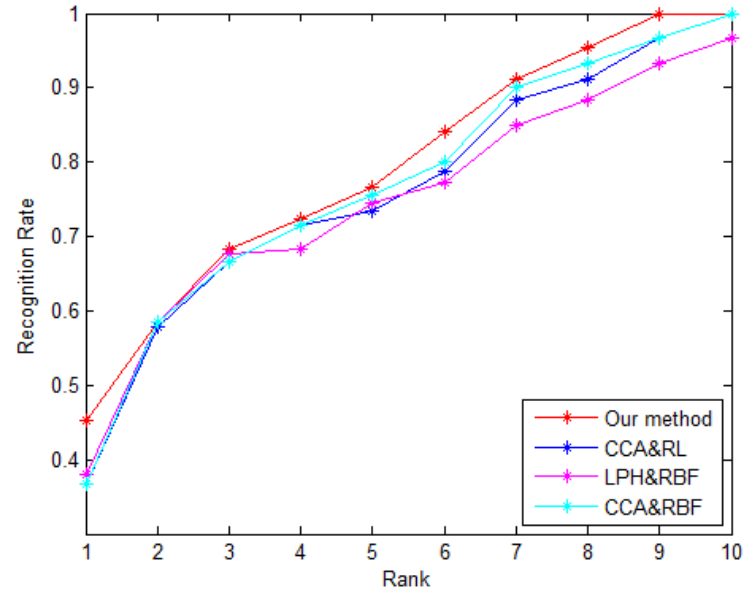

(b)

Fig.2. Experiments results for the Yale face database. (a) Recognition results with different feature dimensions from 0 to 50; (b) Cumulative Match Characteristic(CMC) Curve.

Considering of the computation time and the curve trend, we choose 50 for the largest dimension. In Fig.2 (a), our method, the red line represents, can get higher recognition rate when the dimension is greater than 5. Comparing to other methods, our method with 30 dimensions achieves the highest recognition rate of 0.8400 . When the dimension is too high, the feature will contain more invalid information. So the recognition rate does not always increase along with the dimension.

According to the dimensions changed above, Fig.2(b) shows the cumulative match characteristic(CMC) curve with different training number. As shown in this figure, our method performs best at any rank number. Comparing to the two-step method CCA\&LPH, our method gets the chemical fusion different from the physical fusion of the two-step method.

ORL face database for recognition. In this face database, the training mumble is five, and the other five face images are testing images. Fig.3(a) shows the recognition results with different feature dimensions. In this figure, our method, achieves the highest recognition rate of 0.9750 at the dimension of 35 . When the dimension rises, the recognition rate decreases slowly. When the dimension is greater than 10 , the recognition rate rises slowly. Comparing to other methods, the recognition rate of our method is a little higher than other methods. Comparing to the experiment of Yale database, the gap between our method and other methods is small. It is because that the recognition rate of every method is already high, so the upside potential is limited.

Fig.3(b) shows the CMC curve for ORL face database. In this figure, the recognition rate of our method is significantly higher than other methods when the dimension changes from 5 to 25 . There is a strange phenomenon that the recognition rate of our method has a little decrease when the rank is from 6 to 7 . It shows that our method is stable. When the number of training samples achieves a certain amount, the recognition rate will change slowly and unpredictably. 


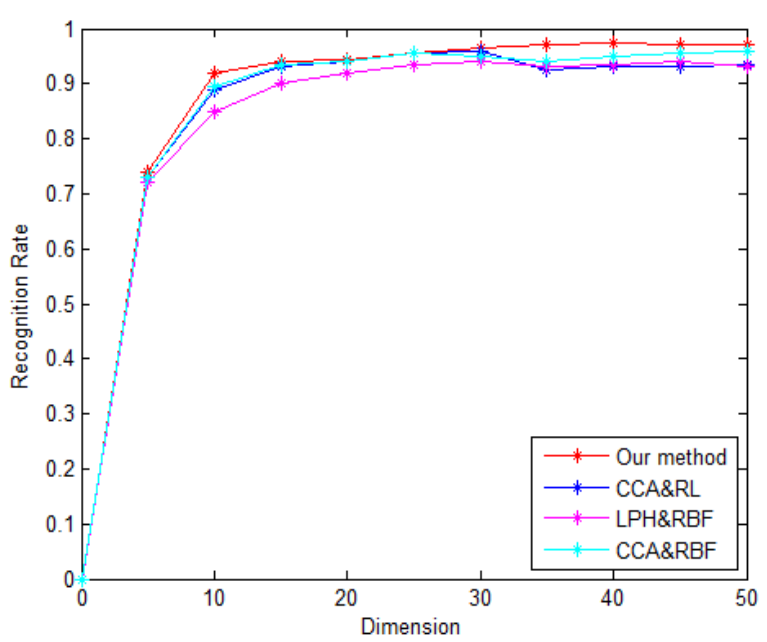

(a)

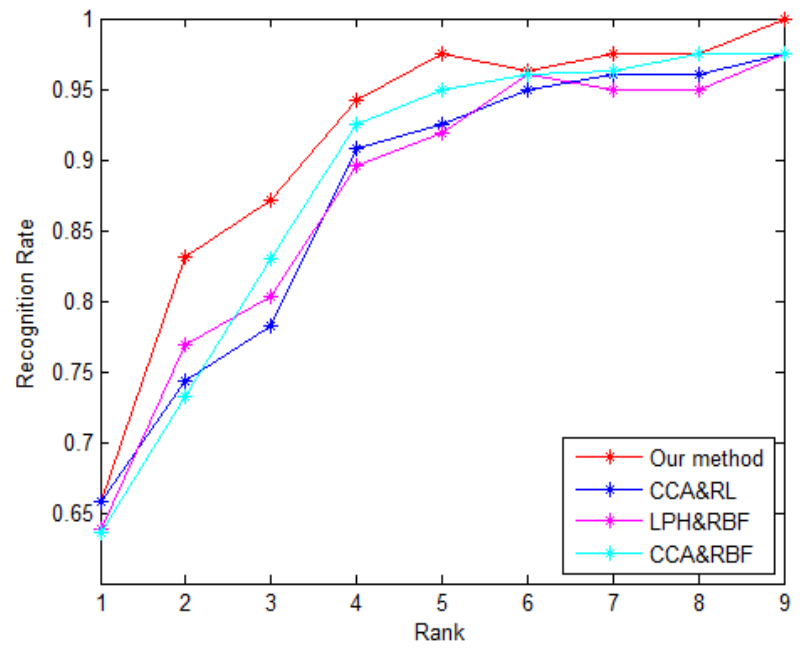

(b)

Fig.3. Experiments results for the ORL face database. (a) Recognition results with different feature dimensions from 0 to 50; (b) Cumulative Match Characteristic(CMC) Curve.

\section{Conclusions and Future Work}

In this paper, SCCA was applied to find the projection subspaces where HR and LR face images had the maximum similarity. To construct the mapping relationship between the coherent features, we used the relationship learning method. Comparing to other SR methods, our method has a higher recognition rate despite the variations of lighting, expression and pose and enhances the robustness to local variants. But relationship learning is an iterative process, which needs more computing. So it is a method with higher recognition rate at the expense of more computation time.

To balance the recognition rate and computation time, we will seek a new solution without iterative to Eq. 6. The second expanding work is to use kernel SCCA to improve recognition rate.

\section{References}

[1] Jian Pu, Jun-pin Zhang, Hua Huang. A survey of super resolution algorithms. Journal of Shandong University (Engineering Science), 2009, 39(1):1-7.

[2] Hong Quan, Chen Song-can, Ni Xue-lei. Sub-pattern canonical correlation anaylsis with application in face recognition. Acta Automatica Sinica, 2008, 34(1):21-30.

[3] Liang Sun, Shui-wang Ji, Jie-ping Ye. A least squares formulation for canonical correlation analysis. International Conference on Machine Learning, 2008, 1024-1031.

[4] Hou Shu-Dong, Sun Quan-Sen, Xia De-Shen. Supervised locality preserving canonical correlation analysis algorithm. PR\&AI, 2012, 25(1):143-151.

[5] Wilman, W.W.Zou, Pong C.Yuen. Very low resolution face recognition problem. IEEE Transactions on Image Processing, 2012, 21(1):327-341.

[6] Hua Huang, Huiting He, Xin Fan. Super-resolution of human face image using canonical correlation ananlysis. Pattern Recognition, 2010, 43(7):2532-2543.

[7] Hua Huang, Huiting He. Super-resolution method for face recognition using nonlinear mappings on coherent features. IEEE transactions on neural network, 2011, 22(1):121-130.

[8] Yue-ting Zhuang, Jian Zhang, Fei Wu. Hallucinating faces: LPH superresolution and neighbor reconstruction for residue compensation. Pattern Recognition, 2007, 40(11):3178-3194. 